\title{
ON A MATHEMATICAL MODEL OF THE PRODUCTIVITY INDEX OF A WELL FROM RESERVOIR ENGINEERING*
}

\author{
AKIF IBRAGIMOV ${ }^{\dagger}$, DINARA KHALMANOVA ${ }^{\ddagger}$, PETER P. VALKO ${ }^{\S}$ AND \\ JAY R. WALTON ${ }^{\ddagger}$
}

\begin{abstract}
Motivated by the reservoir engineering concept of the productivity index of a producing oil well in an isolated reservoir, we analyze a time dependent functional, diffusive capacity, on the solutions to initial boundary value problems for a parabolic equation. Sufficient conditions providing for time independent diffusive capacity are given for different boundary conditions. The dependence of the constant diffusive capacity on the type of the boundary condition (Dirichlet, Neumann, or third boundary condition) is investigated using a known variational principle and confirmed numerically for various geometrical settings. An important comparison between two principal constant values of a diffusive capacity is made, leading to the establishment of criteria when the so-called pseudo-steady-state and boundary-dominated productivity indices of a well significantly differ from each other. The third boundary condition is shown to model the thin skin effect for the constant wellbore pressure production regime for a damaged well. The questions of stabilization and uniqueness of the time independent values of the diffusive capacity are addressed. The derived formulas are used in numerical study of evaluating the productivity index of a well in a general three-dimensional reservoir for a variety of well configurations.
\end{abstract}

Key words. parabolic equation, productivity index, diffusive capacity, time-invariant, skin

AMS subject classification. $76 \mathrm{~S} 05$

DOI. $10.1137 / 040607654$

1. Introduction. In many applied problems, where the modeled processes are, in general, transient, it is important to define such functionals on the solutions, which are, in a sense, time invariant. Existence of such property is important from both practical and theoretical points of view. An important such example to petroleum reservoir engineering, the productivity index (PI), is studied here.

It was long ago observed by petroleum engineers that if a bounded reservoir is depleted by a well, then the ratio of the flow rate to the pressure drawdown (the pressure drop between the reservoir and the wellbore) stabilizes to a constant value. This constant value seems to depend only on the geometrical and hydrodynamical characteristics of the reservoir. In particular, it appears to be independent of the pressure drawdown in the reservoir or the flow rate from the well [23].

The first concise description of this fact was formulated in the classical book by Muskat [23]. The ratio of the rate of flow from the well to the difference between the average pressure on the wellbore and the average pressure in the reservoir is called the productivity index of the well [23]. There are two idealized production regimes considered most frequently for the purpose of analysis in engineering practice: the well can be produced either with a constant flow rate or with a constant wellbore

\footnotetext{
* Received by the editors April 30, 2004; accepted for publication (in revised form) October 19, 2004; published electronically August 3, 2005.

http://www.siam.org/journals/siap/65-6/60765.html

${ }^{\dagger}$ Department of Mathematics and Statistics, Texas Tech University, Lubbock, TX 79409-1042 (akif.ibraguimov@ttu.edu).

${ }^{\ddagger}$ Department of Mathematics, Texas A\&M University, MS 3368, College Station, TX 77840 (dkhalmanova@engr.psu.edu, jwalton@math.tamu.edu).

$\S$ Department of Petroleum Engineering, Texas A\&M University, College Station, TX 77840 (p-valko@tamu.edu).
} 
pressure. In a bounded reservoir depleted in either of the two regimes, the PI of a well stabilizes and remains constant in a long time asymptote.

To analyze the productivity of the well we consider three initial boundary value problems (IBVPs) that correspond to current engineering practice. However, while two of the formulated problems corresponding to the constant pressure production regime are well-posed, the problem modeling the regime with a constant rate of production is ill-posed in the sense of nonuniqueness of solution.

Field operations often reduce the permeability of the region adjacent to the wellbore - the so-called skin zone. Disregarding the skin effect leads to overestimation of the PI of the damaged well $[30,15]$. One of the IBVPs considered in this article models the skin effect in the constant pressure production regime.

The objective for this paper is to build a rigorous mathematical frame for studying the PI. In this respect, it proves useful to introduce the concept of diffusive capacity for a well-reservoir system. The diffusive capacity is an integral type characteristic of the solution of an IVBP. To address the issue of nonuniqueness of solution of the illposed IBVP, we impose restrictions defining a class of solutions in which the diffusive capacity is unique. The inflicted restrictions are motivated by physical considerations as well as traditional engineering practice.

An important property of the PI to stabilize with time regardless of the production regime is then analyzed in terms of the diffusive capacity. Sufficient conditions for the diffusive capacity to be time independent are given for different boundary conditions; through a variational approach to studying the diffusive capacities, its dependence on different boundary conditions is revealed. The obtained theoretical results are then illustrated by numerical computations of the constant diffusive capacities for processes with different boundary conditions in various geometrical settings.

1.1. PI of a well in a bounded reservoir. Reservoir engineering approach: Shape factors. Consider a bounded hydrocarbon reservoir with a flowing fluid (oil) and a well produced with either constant wellbore pressure or constant production rate. The PI of a well is defined as [26]

$$
P I(t)=\frac{q(t)}{p_{w}(t)-p_{a}(t)},
$$

where $q(t)$ is the rate of flow from the well, $p_{w}(t)$ is the flowing bottomhole pressure, and $p_{a}(t)$ is the average pressure of the fluid in the reservoir. When the well is produced with a constant wellbore pressure, its value is taken as $p_{w}(t)$ in (1). The concept of the PI of a well facilitates reservoir engineering methods of estimation of the available reserves and, consequently, helps to optimize the recovery efficiency.

About a century ago it was empirically observed that under either of the two recovery regimes, the PI of a well stabilizes and remains almost constant in a long time asymptote [26]. When the PI of a well is constant, the production regimes have traditionally accepted names: the production regime with the constant rate and constant PI is called a pseudo-steady-state (PSS), and the production regime with the constant wellbore pressure and the constant PI is called a boundary-dominated (BD) state.

The first analytical formula for representation of the PI of a well for a PSS regime was obtained by Muskat [23] for an isolated cylindrical reservoir and a given constant production rate on the fully penetrated vertical well. The IBVPs with the constant rate well boundary condition for a number of typical drainage shapes were first solved 
by Matthews, Brons, and Hazebroek in [21] in connection to the analysis of the buildup wellbore pressure after well shut-in. Using the result of Matthews, Brons, and Hazebroek, an approximate formula for a PSS PI (with skin $s$ ) can be written as

$$
J_{\text {Diet } z}=\frac{1}{\frac{1}{2} \ln \frac{4 V}{\gamma C_{A} r_{w}^{2}}+s},
$$

where $V$ is the area of the two-dimensional reservoir (a three-dimensional reservoir with a uniform thickness), $r_{w}$ is the radius of the circular well, and $\gamma$ is Euler's constant. Equation (2) uses the solution for the dimensionless PSS wellbore pressure first derived by Ramey and Cobb in [27]. The values of the so-called shape factor $C_{A}$ were first presented in [6] and are usually referred to as Dietz's shape factors in the petroleum engineering literature. Positive skin captures the damage to the skin zone, while the negative skin was shown to model a stimulated well [12, 15, 4, 8, 14, 19].

The approximate formula (2) is also used to estimate the productivity of a well produced with a constant bottomhole pressure. However, it is known that the BD state PI of a well is, in general, different from the PSS PI. In particular, the empirical evidence is that the PSS PI is always greater than or equal to the BD PI.

In 1998 Wattenbarger and Helmy derived an algorithm and computed the values of shape factors in (2) for the typical shapes of the drainage area for BD state, using a method of images, Laplace transform, and a fundamental relationship between the images in Laplace space of the cumulative production and the production rate. The applicability of (2) is contingent on the method of images - a drainage area to which the method of images can be applied must be of a shape, which, when translated infinitely many times in all directions, can cover the entire two-dimensional plane.

Most solutions for evaluating the PI in three-dimensional reservoirs, i.e., for directionally drilled wells, follow the same principle as the two-dimensional methods in that they are based on a semianalytical solution for a particular case, from which one finds a convenient approximate formula which is then applied to similar reservoir/well configurations. The semianalytical solution is often based on the superposition of analytical solutions for a transient problem in an unbounded reservoir. For the solution of the problem to be unique, additional assumptions must be made. Usually the restrictions are imposed on the distribution of the pressure on the wellbore. Under one such restriction, the wellbore is assumed to have infinite conductivity, i.e., the wellbore pressure is assumed to be constant on the wellbore at each moment of time. Under another restriction the pressure flux through the wellbore surface is constant at all times.

The solution in a bounded reservoir is then expressed in terms of an infinite time dependent series, similar to the technique used in $[21,16]$. Then a comprehensive computing procedure is applied to determine the stabilized values of the time dependent series in the obtained solution [20, 25, 29, 17, 3, 28].

In most cases the methods for computing the PI of a deviated or horizontal well in a three-dimensional reservoir are aimed at obtaining an appropriate value of a shape factor $C_{A}$ and skin factor $s$ in (2). The effects associated with the deviation of the well from a fully penetrated vertical one are included in the skin $s$. A vertical well is called fully penetrated if its penetration length is equal to the thickness of the reservoir. A vertical fully penetrated well corresponds to $s=0$. The effects of the geometry of the external boundaries of the reservoir are included in the shape factor $C_{A}[20,7]$.

As seen from this brief review, the existing methods and techniques of evaluation of the PI impose serious restrictions on the geometry of the reservoir. In particular, 
the vertical dimension of the reservoir has to be small in comparison to its lateral dimensions to allow one to neglect the flow in the vertical direction or include its effect in the geometrical skin, $s_{g}$. Another restriction is due to the use of the method of images, which requires the drainage area shape to be convex and suitable for covering the whole plane when translated infinitely many times.

One should also note that very little attention has been paid to methods for evaluating a BD PI. For instance, all works mentioned above are concerned only with evaluating the PSS PI in three-dimensional reservoirs. In practice, the BD PI values are taken to be equal to the PSS PI, although it has been shown that the difference between these two values of PI can be up to $10 \%$ even for horizontal flow in simple drainage shapes $[13,16]$.

2. Statement of the problem. Let a point in $\mathbb{R}^{n}$ be denoted by $x=\left(x_{1}, \ldots, x_{n}\right)$, $n=2,3$. Let $\Omega$ be an open domain in $\mathbb{R}^{n}$ which is bounded by the two disjoint piecewise smooth surfaces $\Gamma_{w}$ and $\Gamma_{e}$. Let $u(x, t), t \in \mathbb{R}$, be a solution of the equation

$$
\frac{\partial u}{\partial t}=L u
$$

where $L=\nabla \cdot(A(x) \nabla), A$ is a symmetric positive definite matrix with smooth components and $\nabla=\left(\frac{\partial}{\partial x_{1}}, \ldots, \frac{\partial}{\partial x_{n}}\right)$ is the usual gradient operator.

Let $u(x, t)$ be subject to the homogeneous Neumann boundary condition on $\Gamma_{e}$ :

$$
\frac{\partial u}{\partial \vec{\nu}}=(A(x) \nabla u) \cdot \vec{n}=0,
$$

where $\vec{n}$ is the outward normal to $\Gamma_{e}$. On the remaining part of the boundary, $\Gamma_{w}$, three types of boundary conditions will be considered:

(a) constant total flux $\int_{\Gamma_{w}} \frac{\partial u}{\partial \vec{\nu}} d S=-q, q$ being a real positive constant;

(b) constant Dirichlet condition $\left.u\right|_{\Gamma_{w}}=u_{w 2}, u_{w 2}$ being a real positive constant;

(c) mixed boundary condition $\left.\left(\left.\left(u-u_{w 3}\right)\right|_{\Gamma_{w}}+\alpha \frac{\partial u}{\partial \vec{\nu}}\right)\right|_{\Gamma_{w}}=0$, where $\alpha$ and $u_{w 3}$ are real constants, $u_{w 3}>0$.

For simplicity, we assume that the components of the coefficient matrix $A$ and the domain boundary are smooth, so solutions of the IBVPs I, II, and III (stated below) are understood in a classical sense. In (b), $u_{w 2}>0$ is a given constant; in (c), $u_{w 3}>0$ and $\alpha$ are given constants.

This leads to three IBVPs:

Problem I.

$$
\begin{gathered}
L u=\frac{\partial u}{\partial t}, \quad x \in \Omega, \quad t>0, \\
\left.\frac{\partial u}{\partial \vec{\nu}}\right|_{\Gamma_{e}}=0, \\
\int_{\Gamma_{w}} \frac{\partial u}{\partial \vec{\nu}} d S=-q, \\
u(x, 0)=f_{1}(x) .
\end{gathered}
$$

Remark 1. As mentioned in the introduction, Problem I is ill-posed: there are infinitely many solutions. The PI will be modeled as an integral characteristic of a solution and hence will be lacking uniqueness of definition. Therefore, we will 
consider two classes of solutions in each of which the solution is unique up to an additive constant. These two classes will be described in detail in sections 3 and 4 . Each class has a clear physical meaning. The integral characteristic modeling the PI will be shown to be unique in each class.

Problem II.

$$
\begin{gathered}
L u=\frac{\partial u}{\partial t}, \quad x \in \Omega, \quad t>0, \\
\left.\frac{\partial u}{\partial \vec{\nu}}\right|_{\Gamma_{e}}=0 \\
\left.u\right|_{\Gamma_{w}}=u_{w 2} \\
u(x, 0)=f_{2}(x) .
\end{gathered}
$$

Problem III.

$$
\begin{gathered}
L u=\frac{\partial u}{\partial t}, \quad x \in \Omega, \quad t>0, \\
\left.\frac{\partial u}{\partial \vec{\nu}}\right|_{\Gamma_{e}}=0, \\
\left.\left(\alpha \frac{\partial u}{\partial \vec{\nu}}+\left(u-u_{w 3}\right)\right)\right|_{\Gamma_{w}}=0, \\
u(x, 0)=f_{3}(x) .
\end{gathered}
$$

Remark 2. Physically, $u(x, t)$ is interpreted as the fluid pressure in the reservoir, and hence, we will restrict our attention only to positive solutions of Problems I, II, and III. Moreover, a solution to Problem I is not necessarily positive on $\Omega$ for all $t>0$, even if the initial function $f_{1}(x)$ is positive on $\Omega$. It will be shown that for positive $q$, there exists a solution to Problem I which is positive on $\Omega$ for $t \in(0, T)$ for some positive $T$.

Remark 3. The maximum principle for a parabolic equation implies that the solution of Problem II is unique and positive if the initial condition $f_{2}$ is positive on $\Omega$ [10]. The uniqueness, existence, and regularity of the solutions of Problem III with respect to the sign of the coefficient $\alpha$ in the boundary condition on $\Gamma_{w}$ are discussed, for example, in [10]. Formally, Problem III is a generalization of Problem II. However, we consider Problem II separately in light of its importance for applications in the reservoir engineering.

Remark 4 . The obtained results can be extended to a generalized Wiener solution of an IBVP in a locally smooth domain [18]. We will not present it in this work to preserve the original engineering statement of the problem.

2.1. Definition of diffusive capacity. Let us introduce the following notation. If $v$ is a function defined on $\Omega$, then let $\bar{v}_{w}$ and $\bar{v}_{\Omega}$ denote the average of $v$ on $\Gamma_{w}$ and $\Omega$, respectively, defined by

$$
\bar{v}_{w}=\frac{1}{W} \int_{\Gamma_{w}} v d S
$$

and

$$
\bar{v}_{\Omega}=\frac{1}{V} \int_{\Omega} v d x
$$

where $V=$ mes $_{n} \Omega, W=$ mes $_{n-1} \Gamma_{w}$. 
DEFINITION 1. Let $u(x, t)$ be a classical solution [10] of the parabolic equation $L u=\frac{\partial u}{\partial t}$ in $\Omega \times(0, \infty)$ with boundary condition $\left.\frac{\partial u}{\partial \vec{\nu}}\right|_{\Gamma e}=0$ and (a), (b), or (c) on $\Gamma_{w}$. Let $T>0$ be such that $u(x, t)>0$ for all $x \in \Omega$ and $t \in(0, T)$. The diffusive capacity of $\Gamma_{w}$ with respect to $\Gamma_{e}$ (or simply diffusive capacity) corresponding to the solution $u(x, t)$ is the ratio

$$
J(u, t)=\frac{\int_{\Gamma_{w}} \frac{\partial u}{\partial \vec{\nu}} d S}{\bar{u}_{w}-\bar{u}_{\Omega}}
$$

where $t \in(0, T)$.

Remark 5. For fixed boundary and initial conditions in Problem II (III), the diffusive capacity $J(u, t)$ corresponding to the solution $u$ of Problem II (III) is a function of time only. However, for fixed boundary and initial conditions in Problem I, the diffusive capacity $J(u, t)$ is a time dependent functional on the set of solutions $\{u\}$ to Problem I.

Remark 6. The corresponding diffusive capacity corresponding to a solution of Problem III is defined as

$$
J(u, t)=\frac{\int_{\Gamma_{w}} \frac{\partial u}{\partial \vec{\nu}} d S}{u_{w 3}-\bar{u}_{\Omega}} .
$$

Such correction to the general definition is based on the physical assumption that $u_{w 3}$ is an average wellbore pressure, measured inside the wellbore.

In our intended application, $\Omega$ represents a hydrocarbon reservoir with a flowing fluid (oil) with the outer boundary $\Gamma_{e}$ and a well with boundary $\Gamma_{w}$. The outer boundary of the reservoir is assumed impermeable to the flowing fluid. It is assumed that the fluid is slightly compressible and its flow in the reservoir is governed by Darcy's law relating the gradient of pressure in the reservoir to the filtration velocity $[23,26]$. Then $u(x, t)$ corresponds to the pressure in the reservoir and the three types of boundary conditions specified on the well $\Gamma_{w}$ correspond to different recovery regimes. Boundary condition (a) models the recovery regime with constant production rate, (b) models the recovery regime with constant wellbore pressure, and (c) models the constant wellbore pressure regime of production from a well with nonzero skin [26]. The initial conditions $f_{1}, f_{2}$, and $f_{3}$ take on a meaning of the pressure distribution in the reservoir $\Omega$; hence, we will require that $f_{i} \geq 0$ on $\Omega, i=1,2,3$. IBVP III will be discussed in greater detail in section 5 . The diffusive capacity $J(u, t)$ takes on the meaning of the PI of the well.

3. Time independent diffusive capacity. In this section we show that for each of the IBVP (I, II, and III) there exist initial distributions $f_{1}(x), f_{2}(x)$, and $f_{3}(x)$, respectively, such that the diffusive capacity with respect to the corresponding problem is constant [16]. For Problem I, we describe the class of solutions to IBVP I on which the diffusive capacity takes a unique value. In the last subsection, the time independent values of the diffusive capacity for Problems I, II, and III are compared to each other.

3.1. IBVP I. All solutions of Problem I, for which the diffusive capacity is independent of time, possess the following property.

Remark 7. If $u(x, t)$ is a solution of Problem I and $J(u, t)=J(u)$ is constant for all $t>0$, then there exist real constants $C$ and $B$ such that

$$
\bar{u}_{w}=\frac{1}{W} \int_{\Gamma_{w}} u d S=C+B t .
$$


This can be seen from the following argument. From the definition of the diffusive capacity $(5)$, it follows that $\bar{u}_{w}=-\frac{q}{J(u)}+\bar{u}_{\Omega}$. Hence, $\frac{\partial \bar{u}_{w}}{\partial t}=\frac{\partial \bar{u}_{\Omega}}{\partial t}$. The divergence theorem implies that

$$
\frac{\partial \bar{u}_{\Omega}}{\partial t}=\frac{1}{V} \int_{\Omega} L u d x=\frac{1}{V} \int_{\Gamma_{w}} \frac{\partial u}{\partial \vec{\nu}} d S
$$

Consequently,

$$
\frac{\partial \bar{u}_{w}}{\partial t}=-\frac{q}{V}
$$

from which (7) easily follows.

The "infinite conductivity of the well" assumption asserts that at each instant of time, the pressure on the wellbore is constant. Together with the latter remark, this motivated us to study the diffusive capacity on the class of solutions of Problem I, defined by $\Upsilon=\{u \mid \exists C$ and $B$ are constants, such that $u(x, t)=C+B t$ for $x \in$ $\Gamma_{w}$ and for $\left.t \geq 0\right\}$.

Proposition 1. Problem I has a unique solution in class $\Upsilon$.

Proof. Assume that $u \in \Upsilon$ and $v \in \Upsilon$ are solutions of Problem I. Let $C_{1}, B_{1}, C_{2}$, and $B_{2}$ be such that for $t>0$,

$$
\left.u(x, t)\right|_{\Gamma_{w}}=C_{1}+B_{1} t
$$

and

$$
\left.v(x, t)\right|_{\Gamma_{w}}=C_{2}+B_{2} t .
$$

Then the difference $g(x, t)=u(x, t)-v(x, t)$ is the solution of the following IBVP:

$$
\begin{gathered}
L g=\frac{\partial g}{\partial t}, \quad x \in \Omega, \quad t>0, \\
\left.\frac{\partial g}{\partial \vec{\nu}}\right|_{\Gamma_{e}}=0, \\
\left.g\right|_{\Gamma_{w}}=\left(C_{1}-C_{2}\right)+\left(B_{1}-B_{2}\right) t, \\
g(x, 0)=0 .
\end{gathered}
$$

In addition,

$$
\int_{\Gamma_{w}} \frac{\partial g}{\partial \vec{\nu}}=0 .
$$

Condition (13) immediately implies that $C_{1}=C_{2}$.

The function $h=\frac{\partial g}{\partial t}$ is a solution of the following problem:

$$
\begin{gathered}
L h=\frac{\partial h}{\partial t}, \quad x \in \Omega, \quad t>0, \\
\left.\frac{\partial h}{\partial \vec{\nu}}\right|_{\Gamma_{e}}=0
\end{gathered}
$$




$$
\begin{gathered}
\left.h\right|_{\Gamma_{w}}=B_{1}-B_{2}, \\
h(x, 0)=\frac{\partial g}{\partial t}(x, 0) .
\end{gathered}
$$

In addition, from the boundary condition on $\Gamma_{w}$ of Problem I and the divergence theorem it follows that for $t>0$,

$$
\int_{\Omega} h d x=\frac{\partial}{\partial t} \int_{\Omega} g d x=\int_{\Omega} L g d x \equiv 0
$$

As a solution of the parabolic equation (15) with the Dirichlet condition (17) on one part of the boundary $\partial \Omega$ and Neumann condition (16) on the remaining part of $\partial \Omega$, $h$ will converge to a constant $B_{1}-B_{2}$ on $\Omega$ as $t \rightarrow \infty$ [18]. Together with condition (19) this implies that

$$
\left(B_{1}-B_{2}\right) V=\lim _{t \rightarrow \infty} \int_{\Omega} h(x, t) d x=0 .
$$

Thus, $u=v$.

For purposes that will become clear from Proposition 2, let us introduce the following auxiliary steady-state boundary value problem. Let $u_{1}(x)$ be such that

$$
\begin{gathered}
L u_{1}=-\frac{1}{V}, \\
\left.u_{1}\right|_{\Gamma_{w}}=0, \\
\left.\frac{\partial u_{1}}{\partial \vec{\nu}}\right|_{\Gamma_{e}}=0 .
\end{gathered}
$$

Then the following proposition gives a sufficient condition providing for time independent unique diffusive capacity $J(u, t)=J(u)$.

Proposition 2. If the initial condition in Problem $\mathrm{I}$ is given by $f_{1}(x)=q u_{1}(x)+$ $C$ where $u_{1}$ is the solution of (21)-(23) and $C$ is an arbitrary constant such that $f_{1}(x)>0$ for all $x \in \Omega$, then the diffusive capacity corresponding to a solution $u \in \Upsilon$ of Problem I is independent of time and determined by

$$
J_{I}:=J(u, t)=\frac{V}{\int_{\Omega} u_{1}(x) d x} .
$$

Proof. Let the initial condition in Problem I be $f_{1}(x)=q u_{1}(x)$ and

$$
u(x, t)=q u_{1}(x)-\frac{q}{V} t .
$$

By virtue of the divergence theorem,

$$
\int_{\Gamma_{w}} \frac{\partial u}{\partial \vec{\nu}} d S=-q
$$

Consequently, $u$ is a solution of IBVP I with the initial distribution $f_{1}(x)=q u_{1}(x)$. 
Note that $u$, defined by (25), belongs to class $\Upsilon$. In addition, it is clear that the diffusive capacity $J(u, t)$ on $u(x, t)$ is constant and is given by

$$
J(u, t)=\frac{V}{\int_{\Omega} u_{1}(x) d x}=J_{\mathrm{I}}
$$

Remark 8. Function $u$, defined by (25), is positive on $\Omega$ only for $t \in(0, T)$, where

$$
T=\frac{\min _{x \in \Omega} u_{1}(x)}{V} .
$$

Solutions of Problem I represent the pressure distribution in the reservoir at time $t$; hence, we are interested in the positive on $\Omega$ solutions only. Therefore, the diffusive capacity (as a model of a PSS PI) $J(u, t)=J_{\mathrm{I}}$ is defined only for $t \in(0, T)$, where $T$ is given by (26).

The necessary condition for the time independent diffusive capacity on the solutions of Problem I in class $\Upsilon$ is given by the following proposition.

Proposition 3. If the diffusive capacity $J(u, t)$, corresponding to a solution $u \in \Upsilon$ of Problem $\mathrm{I}$, is constant for all $t>0$, then

$$
\int_{\Omega}\left(u(x, 0)-q u_{1}(x)\right) d x+C^{*}=0
$$

where constant $C^{*}$ is independent of $q$ and $u_{1}$ is the solution of the problem (21)-(23).

Proof. Let $u \in \Upsilon$ be a solution of Problem I such that $J(u, t)=J(u)$ is constant for all $t>0$. Let

$$
g(x, t)=u(x, t)-\left(q u_{1}(x)-\frac{q}{V} t\right) .
$$

There exist constants $C$ and $B$ such that $\left.u\right|_{\Gamma_{w}}=C+B t$. Moreover, by (9), $B=-\frac{q}{V}$. Hence, $g$ is a solution of the problem

$$
\begin{gathered}
L g=\frac{\partial g}{\partial t}, \quad x \in \Omega, \quad t>0 \\
\left.\frac{\partial g}{\partial \vec{\nu}}\right|_{\Gamma_{e}}=0 \\
\left.g\right|_{\Gamma_{w}}=C, \\
g(x, 0)=u(x, 0)-q u_{1}(x) .
\end{gathered}
$$

In addition, $g$ is subject to the following condition:

$$
\int_{\Gamma_{w}} \frac{\partial g}{\partial \vec{\nu}} d S=0
$$

As a solution of the parabolic equation (29) with the boundary conditions (30) and (31), $g(x, t) \rightarrow C$ as $t \rightarrow \infty$. Together with (33), the latter implies that $\bar{g}_{\Omega}=C$ for all $t>0$. Therefore, $\int_{\Omega} g(x, 0) d x=\int_{\Omega}\left(u(x, 0)-q u_{1}(x)\right) d x=C V=C^{*}$.

Remark 9. By Proposition 3, the initial distribution providing for the time independent diffusive capacity is unique up to an additive function of zero average on $\Omega$ and an additive constant independent of the geometry of the domain or boundary conditions.

Remark 10. The integral of the solution of Problem I at $t=0$ represents the initial reserves in the reservoir [9, 2]. The main physical consequence of Proposition 3 is that the diffusive capacity as a model of the PI uniquely determines the average initial amount of the reserves in the reservoir. 


\subsection{IBVP II. Let}

$$
\begin{gathered}
\frac{\partial u_{2}}{\partial t}=L u_{2}, \\
\left.\frac{\partial u_{2}}{\partial \vec{\nu}}\right|_{\Gamma_{e}}=0, \\
\left.u_{2}\right|_{\Gamma_{w}}=0, \\
u_{2}(x, 0)=f_{2}(x)-u_{w 2} .
\end{gathered}
$$

Obviously, $u(x, t)=u_{2}(x, t)+u_{w 2}$ solves Problem II. Then the diffusive capacity for Problem II can be expressed in terms of $u_{2}(x, t)$, namely,

$$
J(u, t):=J\left(u_{2}, t\right)=\frac{\int_{\Gamma_{w}} \frac{\partial u_{2}}{\partial \vec{\nu}} d S}{-\frac{1}{V} \int_{\Omega} u_{2}(x, t) d x} .
$$

Consider the related Sturm-Liouville problem for the elliptic operator $L$ and the first eigenpair of the latter; i.e., let $\lambda_{0}$ and $\phi_{0}(x)$ be the first eigenvalue and first eigenfunction, respectively, of the problem

$$
\begin{gathered}
L \phi_{0}=-\lambda_{0} \phi_{0}, \\
\left.\phi_{0}\right|_{\Gamma_{w}}=0, \\
\left.\frac{\partial \phi_{0}}{\partial \vec{\nu}}\right|_{\Gamma_{e}}=0 .
\end{gathered}
$$

Let $u_{2}(x, t)$ be a solution of the IBVP $(34)-(37)$ with the initial distribution $u_{2}(x, 0)$ equal to $\phi_{0}(x)$. Then $u_{2}(x, t)=\phi_{0}(x) e^{-\lambda_{0} t}$ is a solution of the IBVP (34)-(37). The diffusive capacity is constant and is equal to

$$
J_{\mathrm{II}}:=J\left(u_{2}, t\right)=\frac{\lambda_{0} \int_{\Omega} \phi_{0}(x) d x e^{-\lambda_{0} t}}{\frac{1}{V} \int_{\Omega} \phi_{0}(x) d x e^{\lambda_{0} t}}=\lambda_{0} V .
$$

This leads to the next proposition.

Proposition 4. If the initial condition of Problem II is given by $f_{2}(x)=\phi_{0}(x)+$ $u_{w 2}$, where $\phi_{0}$ is the eigenfunction of problem (39)-(41) corresponding to the minimal eigenvalue $\lambda_{0}$, then the diffusive capacity on the solution $u$ of Problem II is constant and is given by

$$
J(u, t)=J_{\mathrm{II}}=\lambda_{0} V .
$$

In fact, the diffusive capacity is constant provided that the initial distribution $u_{2}(x, 0)$ is equal to any eigenfunction $\phi_{i}(x), i=1,2 \ldots$. However, only the eigenfunction corresponding to the minimal eigenvalue does not change sign on $\Omega$; therefore, in terms of the pressure distribution in the hydrocarbon reservoir, $\phi_{0}(x)$ is the only physically realistic initial distribution. 
3.3. IBVP III. Let $u_{3}(x, t)=u(x, t)-u_{w 3}$, where $u$ solves (III) and $u_{w 3}$ is the given average value of $u$ on $\Gamma_{w}$ (see Remark 6). Then $u_{3}(x, t)$ is a solution of the reduced problem

$$
\begin{gathered}
L u_{3}=\frac{\partial u_{3}}{\partial t}, \\
\left.\frac{\partial u_{3}}{\partial \vec{\nu}}\right|_{\Gamma_{e}}=0, \\
\left.\left(\alpha \frac{\partial u_{3}}{\partial \vec{\nu}}+u_{3}\right)\right|_{\Gamma_{w}}=0, \\
u_{3}(x, 0)=f_{3}(x)-u_{w 3} .
\end{gathered}
$$

Diffusive capacity $J(u, t)$ corresponding to Problem III is expressed in terms of $J\left(u_{3}, t\right)$ in the following way:

$$
J(u, t)=J\left(u_{3}, t\right)=\frac{\int_{\Gamma_{w}} \frac{\partial u_{3}}{\partial \vec{\nu}} d S}{-\frac{1}{V} \int_{\Omega} u d x} .
$$

Physically, the Robin boundary condition on $\Gamma_{w}$ in Problem III corresponds to production from a well with a thin-skin zone with constant wellbore pressure (constant $\left.\left.\bar{u}_{3}\right|_{\Gamma_{w}}\right)[26]$. A sufficient condition for the diffusive capacity to be constant is similar to that for Problem II.

In particular, consider the related Sturm-Liouville problem. Let $\lambda_{k}^{\alpha}$ and $\phi_{k}^{\alpha}(x)$ be an eigenpair of the problem

$$
\begin{gathered}
L \phi_{k}^{\alpha}=-\lambda_{k}^{\alpha} \phi_{k}^{\alpha}, \\
\left.\frac{\partial \phi_{k}^{\alpha}}{\partial \vec{\nu}}\right|_{\Gamma_{e}}=0, \\
\phi_{k}^{\alpha}+\left.\alpha \frac{\partial \phi_{k}^{\alpha}}{\partial \vec{\nu}}\right|_{\Gamma_{w}}=0 .
\end{gathered}
$$

Here, the superscript $\alpha$ is intended to emphasize that the solution and, hence, the diffusive capacity of Problem III depend on the value of parameter $\alpha$. This dependence will be analyzed in subsequent sections. Let $u_{3}(x, t)$ be a solution of the IBVP (43)(46) with the initial distribution $u_{3}(x, 0)=\phi_{k}^{\alpha}(x)$. Then $u_{3}(x, t)=\phi_{k}^{\alpha}(x) e^{-\lambda_{k}^{\alpha} t}$ solves (43)-(46) and the diffusive capacity is time independent.

When parameter $\alpha$ in Problem III is positive, then the minimal eigenvalue $\lambda_{0}^{\alpha}$ is positive and the corresponding eigenfunction $\phi_{0}^{\alpha}(x)$ does not change sign on $\Omega$.

In section 5 we will show that the boundary condition on $\Gamma_{w}$ of Problem III models skin effect for a damaged well produced with a constant wellbore pressure. As mentioned in section 1, the production from a stimulated well is modeled by a negative skin factor $s$; therefore, we will analyze the behavior of the diffusive capacity on the solutions of Problem III for negative values of parameter $\alpha$. The latter case will be discussed in more detail in section 5. For the purposes of this section, it is 
sufficient to note that when $\alpha<0$, the minimal eigenvalue and hence the constant diffusive capacity may be negative. Negative PI is an indication of injection into the well; therefore, to avoid the contradiction, our attention will be restricted to positive eigenvalues only. The analysis of the first eigenfunction will be given in section 5 .

Regardless of the sign of $\alpha$, let $\lambda_{0}^{\alpha}$ be the first nonnegative eigenvalue. If the initial distribution in (43)-(46) is equal to the corresponding eigenfunction, the constant diffusive capacity is given by

$$
J_{\mathrm{III}}(\alpha):=J\left(u_{3}, t\right)=\lambda_{0}^{\alpha} V .
$$

Therefore, we have shown the following proposition.

Proposition 5. If the initial condition of Problem III is given by $f_{3}(x)=$ $\phi_{0}^{\alpha}(x)+u_{w 3}$, where $\phi_{0}^{\alpha}$ is the eigenfunction of problem (48)-(50) corresponding to the minimal positive eigenvalue $\lambda_{0}^{\alpha}$, then the diffusive capacity on the solution $u$ of Problem III is constant and is given by

$$
J(u, t)=J_{\mathrm{III}}(\alpha)=\lambda_{0}^{\alpha} V .
$$

3.4. Comparison of the time independent diffusive capacities for Problems I, II, and III. The steady-state auxiliary problem (21)-(23) introduced earlier has a convenient variational formulation which facilitates deriving an important relation between the time independent diffusive capacities of $\Gamma_{w}$ with respect to $\Gamma_{e}$ in $\Omega$.

Assume that solutions of Problems I, II, and III satisfy the conditions in Propositions 2, 4, and 5, respectively. Then the diffusive capacities for Problems I, II, and III $\left(J_{\mathrm{I}}, J_{\mathrm{II}}\right.$, and $\left.J_{\mathrm{III}}(\alpha)\right)$ are time independent and their values are given by $(24),(42)$, and (51), respectively.

Let $H^{1,2}(\Omega)$ be the Sobolev space [1]. Denote by $H^{1,2}\left(\Omega, \Gamma_{w}\right)$ the closure in the $H^{1,2}(\Omega)$ norm of smooth functions that vanish on $\Gamma_{w}$, and denote by $H^{1,2}\left(\Omega, \Gamma_{w}, \alpha\right)$ the closure in the $H^{1,2}(\Omega)$ norm of smooth functions such that $\left.\left(u+\alpha \frac{\partial u}{\partial \vec{\nu}}\right)\right|_{\Gamma_{w}}=0$ [1].

The following are well-known variational principles yielding the first eigenvalues $\lambda_{0}$ and $\lambda_{0}^{\alpha}$ of the problems (39)-(41) and (48)-(50), respectively (see [5]):

$$
\begin{gathered}
\lambda_{0}=\inf _{\substack{0 \\
u \in H^{1,2}\left(\Omega, \Gamma_{e}\right)}} \frac{\int_{\Omega} A \nabla u \cdot \nabla u d x}{\int_{\Omega} u^{2} d x}, \\
\lambda_{0}^{\alpha}=\inf _{\substack{0 \\
u \in H^{1,2}\left(\Omega, \Gamma_{w} \alpha\right)}} \frac{\int_{\Omega} A \nabla u \cdot \nabla u d x+\frac{1}{\alpha} \int_{\Gamma_{w}} u^{2} d S}{\int_{\Omega} u^{2} d x} .
\end{gathered}
$$

These two principles imply that for any positive $\alpha_{1}$ and $\alpha_{2}$ such that (see [5]) $\alpha_{1}>\alpha_{2}$, $\lambda_{0}^{\alpha_{1}}<\lambda_{0}^{\alpha_{2}}$. Moreover, $\lambda_{0}^{\alpha} \nearrow \lambda_{0}$ as $\alpha \searrow 0$. This leads to the next proposition.

Proposition 6. If the initial conditions in Problems II and III are such that $J_{\text {II }}$ and $J_{\mathrm{III}}(\alpha)$ are time independent and $\alpha \searrow 0$, then $J_{\mathrm{III}}(\alpha) \nearrow J_{\mathrm{II}}$.

Another important comparison can be made between the time independent capacities for Problems I and II.

TheOREm 1. If the initial conditions in Problems I and II are such that the diffusive capacities $J_{\mathrm{I}}$ and $J_{\mathrm{II}}$ are time independent, then

$$
J_{\mathrm{II}} \leq J_{\mathrm{I}} \leq C_{\Omega} J_{\mathrm{II}},
$$


where $C_{\Omega}=\frac{\max _{\Omega} \phi_{0}}{\bar{\phi}_{0}}$.

Proof. Let $u_{1} \in \stackrel{\circ}{H}_{2}^{1}\left(\Omega, \Gamma_{w}\right)$ be a solution of the problem (21)-(23). We need to show that

$$
\frac{1}{\int_{\Omega} u_{1}(x) d x} \geq \lambda_{0}
$$

From (52) it follows that

$$
\lambda_{0} \leq \frac{\int_{\Omega}\left(\nabla u_{1}\right) \cdot\left(A \nabla u_{1}\right) d x}{\int_{\Omega} u_{1}^{2} d x}
$$

Using the identity

$$
\nabla \cdot\left(u_{1} A \nabla u_{1}\right)=\left(\nabla u_{1}\right) \cdot\left(A \nabla u_{1}\right)-u_{1} \nabla \cdot\left(A \nabla u_{1}\right),
$$

applying the divergence theorem to the numerator, and making use of (21)-(23), we obtain

$$
\lambda_{0} \leq \frac{1}{V} \frac{\int_{\Omega} u_{1} d x}{\int_{\Omega} u_{1}^{2} d x}
$$

The last inequality can be rewritten as

$$
\lambda_{0} \leq \frac{1}{V} \frac{\left(\int_{\Omega} u_{1} d x\right)^{2}}{\int_{\Omega} u_{1}^{2} d x} \frac{1}{\int_{\Omega} u_{1} d x} .
$$

The first part of (54) now follows from Hölder's inequality.

Let $u_{1}(x)$ be a solution of $(21)-(23)$ and $\phi_{0}$ of (39)-(41). After multiplication of both sides of (21) by $\phi_{0}$, using the symmetry of $A$ in the identity

$$
\left(\nabla \cdot\left(A \nabla u_{1}\right)\right) \phi_{0}=\nabla \cdot\left(\phi_{0} A \nabla u_{1}\right)-\nabla \cdot\left(u_{1} A \nabla \phi_{0}\right)+\nabla \cdot\left(A \nabla \phi_{0}\right) u_{1},
$$

followed by integration over $\Omega$, from the divergence theorem one concludes that

$$
\lambda_{0} \max _{\Omega} \phi_{0} \int_{\Omega} u_{1} d V \geq \lambda_{0} \int_{\Omega} u_{1} \phi_{0} d V=\frac{1}{V} \int_{\Omega} \phi_{0} d V=\bar{\phi}_{0} .
$$

The latter can be recast as the second part of (54), using the positivity of $u_{1}$ and $\phi_{0}$.

Remark 11 . The constant $C_{\Omega}$ is a peak-to-average ratio and has a clear physical meaning [24].

4. Transient diffusive capacity. In section 3 it was shown that the PI of a well in a reservoir is constant for all $t>0$ provided that the pressure distribution at $t=0$ satisfies certain conditions. The PI is known to stabilize in a long time asymptote regardless of the initial pressure distribution. In this chapter we will consider a transient diffusive capacity and investigate questions related to its stabilization. Thus, we will analyze Problems I and II with arbitrary initial conditions. The only restriction that is imposed on the initial conditions $f_{1}$ and $f_{2}$ of Problems I and II, respectively, is motivated by physical considerations: we require that $f_{1}$ and $f_{2}$ be positive smooth functions on $\Omega$. 
4.1. IBVP I: Constant production rate regime. In section 1 it was mentioned that the constant rate regime is usually modeled with one of two assumptions: at each time $t>0$ either the pressure or the pressure flux is assumed to be constant on the wellbore. Proposition 2 shows that the condition of a constant wellbore pressure at each time $t>0$ (infinite conductivity condition) is equivalent to the conditions of the PSS, i.e., the PI of a well is time independent. In this section we will show that the diffusive capacity on the class $\Upsilon$ of solutions of Problem I (defined in section 3) is stable with respect to small perturbations of boundary conditions. Recall that $\Upsilon$ is the class of solutions $u$ of Problem I such that at each time $t>0, u$ is constant on $\Gamma_{w}$. Then the stability of $J$ is established by the following proposition.

Proposition 7. Let $v(x, t)$ be a solution of Problem I such that $v(x, t)=B t+C$ for all $x \in \Gamma_{w}$. Let $u(x, t)$ be a solution of Problem I such that $u(x, t)=B t+$ $C+h(x, t)$ for all $x \in \Gamma_{w}$, where $h(x, t)$ is a smooth, bounded function. For any $\epsilon>0$, there exists $\delta>0$ such that if for all $t>0,|h(x, t)| \leq \delta$ for all $x \in \Gamma_{w}$, then $|J(u, t)-J(v, t)| \leq \epsilon$ for all $t>0$.

Proof. Function $\tilde{v}(x, t)=u(x, t)-v(x, t)$ is a solution of the following problem:

$$
\begin{gathered}
L \tilde{v}=\frac{\partial \tilde{v}}{\partial t}, \quad x \in \Omega, \quad t>0, \\
\left.\frac{\partial \tilde{v}}{\partial \vec{\nu}}\right|_{\Gamma_{e}}=0 \\
\left.\tilde{v}\right|_{\Gamma_{w}}=h(x, t), \\
\tilde{v}(x, 0)=0 .
\end{gathered}
$$

The maximum principle for parabolic equation (60) implies that $|\tilde{v}(x, t)| \leq \delta$ for all $x \in \Omega$ and $t \geq 0$. Since $\int_{\Gamma_{w}} \frac{\partial u}{\partial \nu} d S=\int_{\Gamma_{w}} \frac{\partial u}{\partial \nu} d S=-q$ for $t \geq 0$,

$$
\left|\frac{1}{J(v, t)}-\frac{1}{J(u, t)}\right| \leq \frac{1}{q}\left|\frac{1}{W} \int_{\Gamma_{w}}(u-v) d S+\frac{1}{V} \int_{\Omega}(u-v) d x\right| .
$$

Hence, $\left|\frac{1}{J(v, t)}-\frac{1}{J(u, t)}\right| \leq \delta$.

One should note that $J_{\mathrm{I}}$ is shown to be a PSS PI of a well only for solutions of Problem I that belong to class $\Upsilon$. The extent to which the assumption of the infinite conductivity of the well is realistic for various reservoir-well configurations will be discussed in more detail in section 7 . Below we investigate the question of the uniqueness of the PSS PI. Recall that the PSS PI is a constant value of the diffusive capacity on the solutions to Problem I.

Remark $12 . J_{\mathrm{I}}$ is not necessarily a unique constant value of the diffusive capacity on the solutions to Problem I.

This is established by the following argument. Consider solutions to Problem I with a constant flux on $\Gamma_{w}$; i.e., let $u(x, t)$ be a solution of the following problem:

$$
\begin{gathered}
L u=\frac{\partial u}{\partial t}, \quad x \in \Omega, \quad t>0, \\
\left.\frac{\partial u}{\partial \vec{\nu}}\right|_{\Gamma_{e}}=0,
\end{gathered}
$$




$$
\begin{aligned}
& \left.\frac{\partial u}{\partial \vec{\nu}}\right|_{\Gamma_{w}}=-\frac{q}{W}, \\
& u(x, 0)=f_{1}(x) .
\end{aligned}
$$

The solution to (64)-(67) is given (up to an additive constant) by $u(x, t)=q v-\frac{q}{V} t+$ $h(x, t)$, where $v(x)$ is a solution of the steady-state problem

$$
L v=-\frac{1}{V}, \quad x \in \Omega,
$$

$$
\begin{gathered}
\left.\frac{\partial v}{\partial \vec{\nu}}\right|_{\Gamma_{e}}=0 \\
\left.\frac{\partial v}{\partial \vec{\nu}}\right|_{\Gamma_{w}}=-\frac{1}{W}
\end{gathered}
$$

and $h(x, t)$ is a solution of the corresponding problem with homogeneous boundary conditions:

$$
\begin{gathered}
L h=\frac{\partial h}{\partial t}, \quad x \in \Omega, \quad t>0, \\
\left.\frac{\partial h}{\partial \vec{\nu}}\right|_{\Gamma_{e}}=0 \\
\left.\frac{\partial h}{\partial \vec{\nu}}\right|_{\Gamma_{w}}=0 \\
h(x, 0)=f_{1}(x)-q v(x) .
\end{gathered}
$$

The solution to (71)-(74) is given by $h(x, t)=\sum_{n=0}^{\infty} c_{n} \phi_{n}(x) e^{-\lambda_{n} t}$, where $\phi_{n}(x)$ and $\lambda_{n}$ are solutions of the related Sturm-Liouville problem and $c_{n}$ are the coefficients of the Fourier expansion of $h(x, 0)$ in terms of $\phi_{n}$. The diffusive capacity $J(u, t)$ is given by

$$
J(u, t)=\frac{-q}{\bar{v}_{w}-\bar{v}_{\Omega}+\bar{h}_{w}-\bar{h}_{\Omega}} .
$$

Note that $\bar{v}_{w}$ and $\bar{v}_{\Omega}$ are constant, while $\bar{h}_{w}$ and $\bar{h}_{\Omega}$ are functions of time. Clearly, the difference $\bar{h}_{w}-\bar{h}_{\Omega}=\sum_{n=0}^{\infty} c_{n}\left(\bar{\phi}_{n_{w}}-\bar{\phi}_{n \Omega}\right) e^{-\lambda_{n} t}$ converges to a constant as $t \rightarrow \infty$. Therefore, $J(u, t)$ converges to a constant value $\hat{J}$ as $t \rightarrow \infty$. However, $\hat{J}$ is not necessarily equal to $J_{\mathrm{I}}$.

Henceforth, we do not address the uniqueness of the constant diffusive capacity on the solutions of Problem I and, consequently, of the PSS PI. In the subsequent sections we will refer to $J_{\mathrm{I}}$ as the value of the PSS PI, thus implicitly assuming that the wellbore has an infinite conductivity. 
4.2. IBVP II: Constant wellbore pressure regime. For simplicity, consider the following problem for a parabolic equation. Let $u(x, t)$ be a solution of

$$
\begin{gathered}
L u=\frac{\partial u}{\partial t}, \quad x \in \Omega, \quad t \geq 0, \\
\left.\frac{\partial u}{\partial \vec{\nu}}\right|_{\Gamma_{e}}=0, \\
\left.u\right|_{\Gamma_{w}}=0 \\
u(x, 0)=u_{0}(x),
\end{gathered}
$$

where $u_{0}(x)>0$. Then the diffusive capacity is simply

$$
J(u, t)=V \frac{\int_{\Gamma_{w}} \frac{\partial u}{\partial \vec{\nu}} d S}{\int_{\Omega} u d x} .
$$
ator $L$,

Along with (76)-(79), consider the related Sturm-Liouville problem for the oper-

$$
\begin{gathered}
L \phi_{k}=-\lambda_{k} \phi_{k}, \quad x \in \Omega, \quad t \geq 0, \\
\left.\frac{\partial \phi_{k}}{\partial \vec{\nu}}\right|_{\Gamma_{e}}=0 \\
\left.\phi_{k}\right|_{\Gamma_{w}}=0 .
\end{gathered}
$$

Let $\left\{\phi_{k}(x)\right\}_{k=0}^{\infty}$ be an orthonormal family of solutions of (81)-(83) with respect to the usual inner product in $L^{2}(\Omega)$. Define $d_{k}=\int_{\Omega} \phi_{k}(x) d x$ and $c_{k}=\int_{\Omega} u_{0}(x) \phi_{k}(x) d x$. Then the diffusive capacity can be written as

$$
J(u, t)=V \frac{\sum_{k=0}^{\infty} c_{k} \lambda_{k} d_{k} e^{-\lambda_{k} t}}{\sum_{k=0}^{\infty} c_{k} d_{k} e^{-\lambda_{k} t}} .
$$

The latter can be recast into

$$
J(u, t)=V \lambda_{0}\left[1+\frac{\sum_{k=1}^{\infty} \frac{c_{k}}{c_{0}} \frac{d_{k}}{d_{0}}\left(\frac{\lambda_{k}}{\lambda_{0}}-1\right) e^{-\left(\lambda_{k}-\lambda_{0}\right) t}}{1+\sum_{k=1}^{\infty} \frac{c_{k}}{c_{0}} \frac{d_{k}}{d_{0}} e^{-\left(\lambda_{k}-\lambda_{0}\right) t}}\right] .
$$

Since $\lambda_{0}<\lambda_{1}<\lambda_{3}<\cdots$, as $t \rightarrow \infty, J(u, t) \rightarrow \lambda_{0} V$. This proves the following.

Proposition 8. If $u$ is a solution of IBVP II, then the diffusive capacity $J(u, t)$ converges to the constant value $J_{\mathrm{II}}$ as $t \rightarrow \infty$ for any initial condition $f_{2}$.

In terms of the PI, Proposition 8 can be rephrased in the following way: if a well is produced with a constant wellbore pressure, the PI stabilizes to constant value $J_{\text {II }}$ as $t \rightarrow \infty$ regardless of the initial pressure distribution.

Note that since the initial condition $u_{0}(x)$ is positive on $\Omega, c_{0}>0$ and $d_{0}>0$. From the maximum principle for parabolic equation (76) it follows that $u(x, t) \geq 0$ 
for all $t>0$. Consequently, the denominator in (84), equal to $\int_{\Omega} u(x, t) d x / c_{0} d_{0} e^{-\lambda_{0} t}$, is positive for all $t>0$. Therefore, from (84) follows the next remark.

Remark 13. If in (84) $c_{k} d_{k}>0$ for any $k$, then $J(u, t) \searrow \lambda_{0} V$.

The last observation allows one to analyze several physically important examples of the transient PI in terms of the diffusive capacity on the solutions of the IBVP for a parabolic equation.

Example 1. Suppose that a well is produced with constant rate, the PI is constant, and the well has infinite conductivity. Then the pressure in the reservoir $u(x, t)$ is determined (up to an additive constant) by $u(x, t)=q u_{1}(x)-\frac{q}{V} t$ (see Proposition 2), where $u_{1}(x)$ is a solution of the auxiliary steady-state problem

$$
\begin{gathered}
L u_{1}(x)=-\frac{1}{V}, \quad x \in \Omega, \\
\left.\frac{\partial u_{1}}{\partial \vec{\nu}}\right|_{\Gamma_{e}}=0 \\
\left.u_{1}\right|_{\Gamma_{w}}=0 .
\end{gathered}
$$

Suppose that at some time $t_{0}>0$, the production regime was changed to a constant wellbore pressure production. Then the pressure in the reservoir $u(x, t)$ for $t>t_{0}$ is defined by $u(x, t)=v\left(x, t-t_{0}\right)-\frac{q}{V}\left(t-t_{0}\right)$, where $v(x, t)$ is a solution of the problem

$$
\begin{gathered}
L v(x)=-\frac{\partial v}{\partial t}, \quad x \in \Omega, \quad t>0 \\
\left.\frac{\partial v}{\partial \vec{\nu}}\right|_{\Gamma_{e}}=0 \\
\left.v\right|_{\Gamma_{w}}=0 \\
v(x, 0)=q u_{1}(x) .
\end{gathered}
$$

The diffusive capacity $J(u, t)=J(v, t)$, where $v(x, t)$ is defined by

$$
v(x, t)=\sum_{n=0}^{\infty} c_{k} \phi_{k}(x) e^{-\lambda_{k} t}
$$

where $c_{k}=q \int_{\Omega} u_{1}(x) \phi_{k} d x$. Using integration by parts, we obtain

$$
\int_{\Omega} L u_{1} \phi_{k}=\int_{\Omega} u_{1} L \phi_{k}
$$

Hence,

$$
\frac{1}{V} \int_{\Omega} \phi_{k}=\lambda_{k} \int_{\Omega} u_{1} \phi_{k}
$$




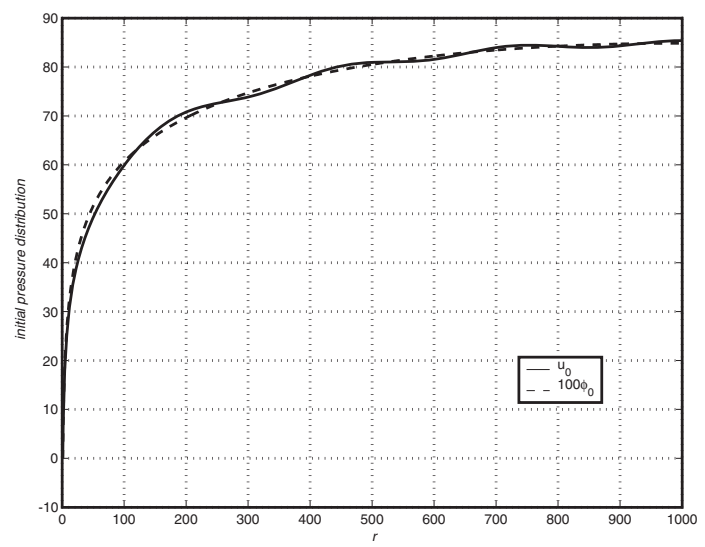

FIG. 1. Radial profile of an initial distribution yielding small diffusive capacity.

Thus, for any $k=1,2 \ldots, d_{k} c_{k}>0$ and (84) implies that $J(u, t) \searrow J_{\mathrm{II}}$. In other words, when the regime of production changes from PSS, i.e., constant flow rate, to constant wellbore pressure, the PI monotonically decreases to the BD PI.

Example 2. For the purpose of analysis it is frequently assumed that at $t=0$ the pressure in the reservoir is distributed uniformly, i.e., $u_{0}(x)=u_{i}$, where $u_{i}$ is a positive constant. Then $c_{k}=u_{i} d_{k}$ and the PI is monotonically decreasing to the BD PI.

Finally, consider an example of the initial pressure distribution yielding the PI which is less than the BD PI. $\lambda_{0} V$.

Example 3. Let $u_{0}(x)=100 \phi_{0}(x)-3 \phi_{1}(x)$. Then the diffusive capacity $J(u, t)<$

An example of such initial distribution for an ideal cylindrical reservoir with vertical fully penetrated well is given in Figure 1, where the radial profile of $u_{0}(r)$ is given. The dimensionless radius of the reservoir is equal to $R_{D}=1000$. Physically this example may be interpreted as follows. Assume that the reservoir has been depleted by a set of wells. Suppose that the old wells are shut down and a new well is drilled and produced. Then the PI of the new well will monotonically increase to the BD PI value.

5. Model of the skin effect. Stabilized production with constant rate is characterized by the PSS PI. When the well is damaged, the value of the PI is less than what is predicted by the model. As described in section 1, such effect is called thinskin effect. To take into account the skin effect, the PSS PI is corrected according to the equation

$$
P I_{P S S, s k i n}=\frac{1}{\frac{1}{P I_{P S S}}+s}
$$

where $s$ is the so-called skin factor or simply skin. The skin factor concept was originally introduced to describe the behavior of damaged wells. Others have extended the idea to stimulated wells which have a higher PI than the PSS PI of an ideal well. In [15] it was shown that a negative skin $s$ corresponds to a stimulated well.

All existing results on modeling the skin effect pertain to the constant rate production regime. In this section it will be shown that for the constant wellbore pressure 
production regime, the skin effect can be modeled by a third boundary condition specified on the well boundary.

5.1. Diffusive capacity for IBVP III in an annulus. Let $u(r, t)$ be a solution of the problem

$$
\begin{gathered}
\frac{\partial}{\partial r}\left(r \frac{\partial u}{\partial r}\right)=\frac{\partial u}{\partial t}, \quad 1<r<R_{D}, \quad t>0 \\
\left.\frac{\partial u}{\partial r}\right|_{r=R_{D}}=0 \\
\left.\left(u+\alpha \frac{\partial u}{\partial r}\right)\right|_{r=1}=0 \\
u(r, 0)=u_{0}(r)
\end{gathered}
$$

Problem (86)-(89) models the axisymmetric flow of oil in an ideal isolated circular reservoir with a perfect circular well situated in the center. Here, $u(r, t)$ is the dimensionless pressure in the reservoir, the dimensionless formation permeability is 1 , and the dimensionless outer radius is equal to $R_{D}$. The dimensionless wellbore radius is equal to 1 . Constant wellbore pressure production is assumed. The thin skin zone adjacent to the well has a permeability below than that of the formation.

We will call a production regime for a well with a thin skin zone characterized by a constant PI a generalized BD state. When $\alpha=0$ (no damaged zone around the well), it is a $\mathrm{BD}$ regime.

Along with problem (86)-(89), consider a related Sturm-Liouville problem:

$$
\begin{gathered}
\frac{\partial}{\partial r}\left(r \frac{\partial \phi_{k}^{\alpha}}{\partial r}\right)=-\lambda_{k}^{\alpha} \partial \phi_{k}^{\alpha}, \quad 1<r<R_{D}, \quad t>0 \\
\left.\frac{\partial \phi_{k}^{\alpha}}{\partial r}\right|_{r=R_{D}}=0 \\
\left.\left(\phi_{k}^{\alpha}+\alpha \frac{\partial \phi_{k}^{\alpha}}{\partial r}\right)\right|_{r=1}=0 .
\end{gathered}
$$

Let $\lambda_{0}^{\alpha}$ be the minimal nonnegative eigenvalue of the problem (90)-(92). If the initial condition $u_{0}(r)=\phi_{0}^{\alpha}$ is the eigenfunction corresponding to $\lambda_{0}^{\alpha}$, then by Propositions 5 and 6 the generalized BD PI is determined by $J_{\mathrm{III}}(\alpha)=\lambda_{0}^{\alpha} V$ and $J_{\mathrm{III}}(0)=J_{\mathrm{II}}$.

In analogy to (85), we define the skin factor $s$ by

$$
s=s(\alpha):=\frac{1}{J_{\mathrm{III}}(\alpha)}-\frac{1}{J_{\mathrm{II}}}=\frac{1}{J_{\mathrm{III}}(\alpha)}-\frac{1}{J_{\mathrm{III}}(0)} .
$$

Positive skin defined by (93) is evidence of a damaged well. By analogy, the generalized BD index of a stimulated well should be greater than the BD index, yielding negative skin $s$. 

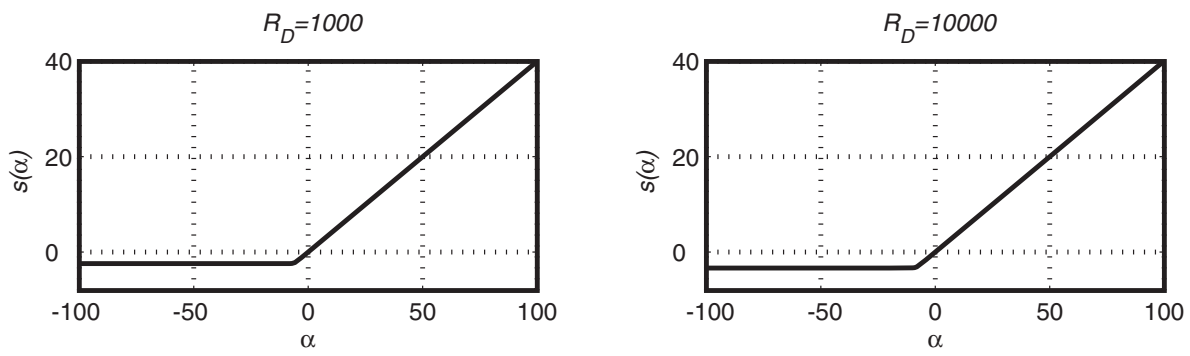

Fig. 2. Graph of $s(\alpha)$ for $R_{D}=1000$ (left panel) and for $R_{D}=10,000$ (right panel).

When $\alpha<0, \lambda_{0}^{\alpha}$ is the first positive eigenvalue. The eigenpair solves known equations involving Bessel functions of the first and the second kind. Using known facts from the theory of Bessel functions, it is not hard to show the following.

Proposition 9. As $\alpha \rightarrow \infty, \lambda_{0}^{\alpha} \rightarrow 0$. As $\alpha \rightarrow-\infty, \lambda_{0}^{\alpha} \rightarrow \lambda_{0}^{(N)}$, where $\lambda_{0}^{(N)}$ is the minimal nontrivial eigenvalue of the following problem:

$$
\begin{gathered}
\frac{\partial}{\partial r}\left(r \frac{\partial u}{\partial r}\right)=\frac{\partial u}{\partial t}, \quad 1<r<R_{D}, \quad t>0 \\
\left.\frac{\partial u}{\partial r}\right|_{r=R_{D}}=0 \\
\frac{\partial u}{\partial r}=0 \\
u(r, 0)=u_{0}(r) .
\end{gathered}
$$

This implies, in particular, that $s(\alpha)$, defined by (93), is bounded from below, since $\lambda_{0}^{(N)}$ is bounded from above. The relation between $s$ and $\alpha$ for $R_{D}=1000$ and $R_{D}=1000$ is shown in Figure 2 for a range of values of $\alpha$. Figure 2 illustrates that when $\alpha>0$, skin $s=\alpha$, i.e., the positive skin can be successfully modeled by the third boundary condition, in perfect agreement with the constant rate case. To analyze the case of $\alpha<0$, additional considerations are necessary.

Eigenfunctions $\phi_{0}^{\alpha}$ corresponding to the minimal positive eigenvalue $\lambda_{0}^{\alpha}$ of the problem (90)-(92) for two sample positive and negative values of $\alpha$ are pictured in Figure 3. As seen in Figure 3, for negative $\alpha$ the corresponding eigenfunction $\phi_{0}^{\alpha}$ changes sign on the interval $1<r<R_{D}$. Recall that the initial condition of the problem (86)-(89) $u_{0}$ is equal to $\phi_{0}^{\alpha}$. Consequently, the sufficient condition for the generalized BD state is such that the initial pressure distribution in the reservoir is not everywhere positive. Thus, a negative value of the skin factor $s$ creates a physical contradiction, and problem (86)-(89) with $\alpha<0$ cannot serve as an appropriate model for a stimulated well. 

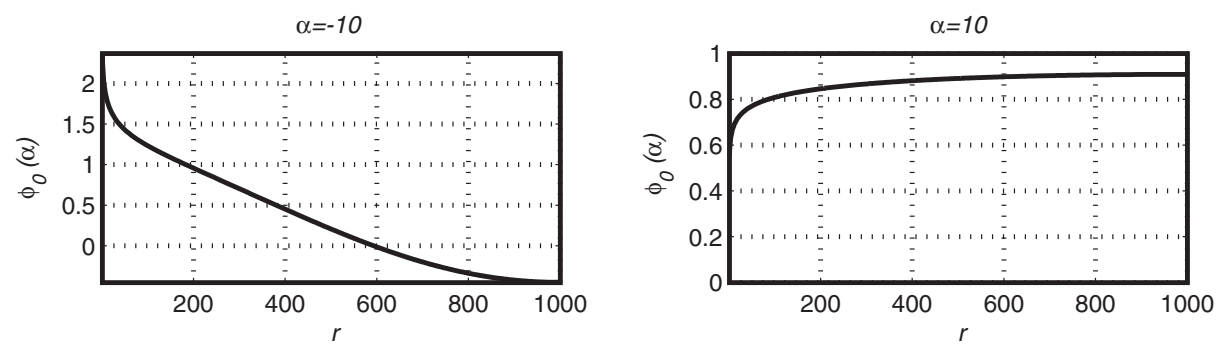

FIG. 3. Eigenfunctions for negative $\alpha$ (left panel) and positive $\alpha$ (right panel). $R_{D}=1000$.

6. PI in a two-dimensional reservoir. In this chapter we present a numerical study of the diffusive capacity/PI in two-dimensional domains. We will restrict our attention to PSS and BD productivity indices only, that is, we will consider only IBVPs I and II.

If the thickness of the reservoir is uniform, then for a fully penetrated vertical well the three-dimensional problem reduces to a two-dimensional one. Since the radius of wellbore is small compared to the dimensions of the reservoir, we can assume that the pressure is uniformly distributed on the wellbore. Therefore, for a two-dimensional problem, the PSS PI is equal to $J_{\text {I }}$ given by $(24)$.

Under the assumption that the reservoir is ideal and the well is perfectly circular, vertical, and fully penetrated, the IBVPs I and II can be formulated in terms of dimensionless variables as follows. Let $\Omega \in \mathbb{R}^{2}$ be the horizontal cross-section of such a reservoir. Let $\{r, \theta\}$ be a polar coordinate system specified on $\Omega$ along with the Cartesian coordinate system $\{x, y\}$. The origins of both coordinate systems are located at the center of the well, which is represented by a circle with equation $r=1$. Let $R_{D}$ be the radius of the circle of the same area as $\Omega$. Then the dimensionless area $V$ of $\Omega$ is equal to $\left(R_{D}^{2}-1\right) / 2$. As before, let $\Gamma_{e}$ denote the exterior boundary of $\Omega$. The auxiliary steady-state problem (21)-(23) and the Sturm-Liouville problem (39)-(41) can be written as

$$
\begin{gathered}
\frac{\partial^{2} u_{1}}{\partial x^{2}}+\frac{\partial^{2} u_{1}}{\partial y^{2}}=-\frac{1}{V}, \\
\left.u_{1}\right|_{r=1}=0 \\
\left.\frac{\partial u_{1}}{\partial \vec{n}}\right|_{\Gamma_{e}}=0
\end{gathered}
$$

and

$$
\begin{gathered}
\frac{\partial^{2} \phi_{0}}{\partial x^{2}}+\frac{\partial^{2} \phi_{0}}{\partial y^{2}}=-\lambda_{0} \phi_{0}, \\
\left.\phi_{0}\right|_{r=1}=0, \\
\left.\frac{\partial \phi_{0}}{\partial \vec{n}}\right|_{\Gamma_{e}}=0,
\end{gathered}
$$


respectively. By Propositions 2 and 4, the values of the PSS and BD PIs are given by the following equations, respectively:

$$
J_{\mathrm{I}}=\frac{V}{\int_{\Omega} u_{1} d x}
$$

and

$$
J_{\mathrm{II}}=\lambda_{0} V
$$

As the first stage, $J_{\mathrm{I}}$ and $J_{\mathrm{II}}$ values were compared to the values obtained by Dietz's equation (2) for domains in which (2) can be applied, that is, for domains with polygonal exterior boundaries: rectangle, triangle, circle, romb, and hexagon. Value $J_{\mathrm{I}}$ was compared to the value of the PSS PI $J_{P S S}$ computed by (2) with the shape factors $C_{A}$ taken from [6] for every considered shape. The constant diffusive capacity $J_{\mathrm{II}}$, given by (105), was compared to the PI $J_{B D}$ computed by (2) with the BD shape factors $C_{A}$ provided in [13]. The results were obtained for two values of the dimensionless radius $R_{D}$ of the drainage area, $R_{D}=1000$ and $R_{D}=10,000$.

The obtained results are not presented here due to limited space, but (104) and (105) closely agree to the corresponding existing formulas. The largest difference between the corresponding values is the one between $J_{\mathrm{I}}$ and $J_{\mathrm{II}}$ in the drainage areas where the well is located far from the center of symmetry of the domain.

As noted, one of the disadvantages of (2) is that it cannot be applied to the drainage area shapes that do not satisfy the requirements of the method of images. On the other hand, (104) and (105) are valid for all drainage area shapes and can be applied to a general reservoir without the usual assumptions of the homogeneity and isotropy of the media. Below we exploit these useful features of the new formulas for PI to analyze its behavior in more complex geometries and for anisotropic media. Then, using the new method we will evaluate the diffusive capacity in domains with more complex geometry, revealing some geometric characteristics of the domain that lead to the nonnegligible difference between $J_{\mathrm{I}}$ and $J_{\mathrm{II}}$.

6.1. PI in domains violating isoperimetric inequality. Theorem 1 of section 3 gives the means to investigate more deeply the effects on the difference between $J_{\mathrm{I}}$ and $J_{\text {II }}$ of the shape of the exterior boundary of the domain. The difference between $J_{\mathrm{I}}$ and $J_{\mathrm{II}}$ is expected to be greater when the constant $C_{\Omega}$ on the right-hand side of inequality (54) is much greater than 1 . The constant $C_{\Omega}$ is, in its turn, determined by the minimal eigenvalue $\lambda_{0}$ and the behavior of the corresponding eigenfunction $\phi_{0}$ of the elliptic problem (101)-(103).

The first eigenpair of the problem is directly related to the geometry of the domain, namely, to the symmetry and curvature of the exterior boundary and the shape of the well boundary. To illustrate the effect of the curvature and the symmetry of the exterior boundary, consider domains in Figure 4 (A) and (B). If the domain does not satisfy the classical isoperimetric inequality, the first eigenvalue of the problem (101)-(103) can be small enough in comparison to $C_{\Omega}$ to make the difference between $J_{\mathrm{I}}$ and $J_{\text {II }}$ significant [22]. It is not hard to show that for $0<\epsilon<1$, both domains pictured in Figure 4 violate the classical isoperimetric inequality [22]. For either shape, the domain parameters $b$ and $\epsilon$ change so that the ratio of the area of the domain to the radius of the well is held constant and corresponds to $R=1000$. The circular well is located in the center of the area. The results of the numerical investigation for domains violating the classical isoperimetric inequality [22] are collected in Table 1. 


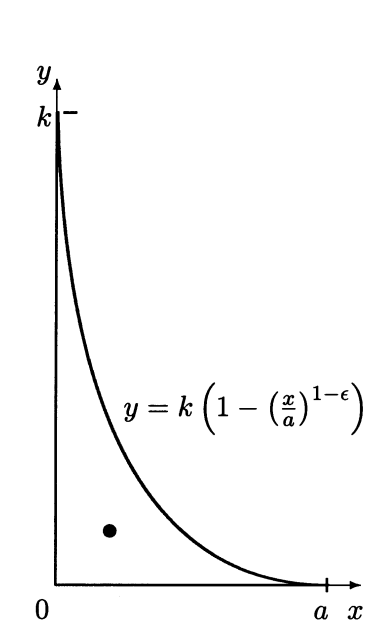

$(A)$

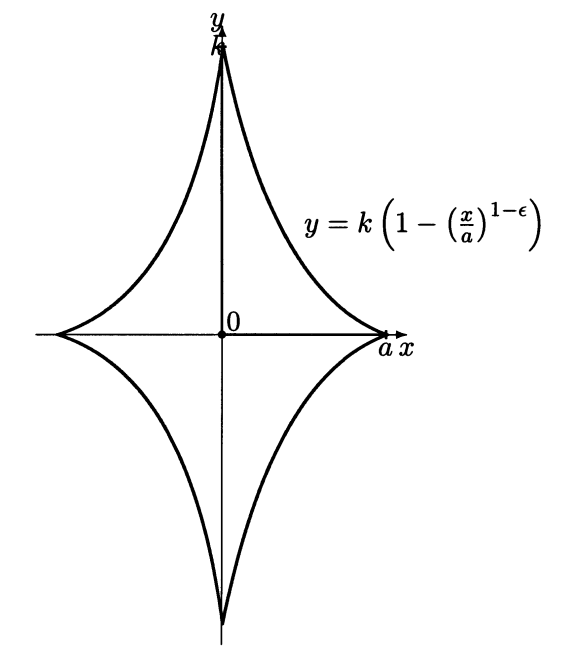

$(B)$

FIG. 4. Domains violating isoperimetric inequality.

TABLE 1

The difference between $J_{\mathrm{I}}$ and $J_{\mathrm{II}}$ in domains violating the isoperimetric inequality.

\begin{tabular}{|l||c|c|c|c||}
\hline Shape & $\epsilon$ & $J_{\mathrm{I}}$ & $J_{\mathrm{II}}$ & $\begin{array}{c}\frac{J_{\mathrm{I}}-J_{\mathrm{II}}}{J_{\mathrm{II}}} \\
\text { percent }\end{array}$ \\
\hline \hline \multirow{3}{*}{ see Figure 4 (A) } & 0.0 & 0.1227 & 0.1065 & 4.69 \\
\cline { 2 - 5 } & 0.4 & 0.0539 & 0.4370 & 23.34 \\
\cline { 2 - 5 } & 0.6 & 0.0137 & 0.0100 & 37.00 \\
\hline \hline \multirow{3}{*}{ see Figure 4 (B) } & 0.8 & 0.0071 & 0.005 & 39.22 \\
\cline { 2 - 6 } & 0.95 & 0.0990 & 0.1222 & 19.00 \\
\hline
\end{tabular}

The symmetrical domain is presented to illustrate the importance of symmetry: the difference between $J_{\mathrm{I}}$ and $J_{\mathrm{II}}$ for a symmetrical domain is significantly less than for a nonsymmetrical domain with the same curvature of the exterior boundary.

7. PI in a three-dimensional reservoir. As described in the introduction, the existing methods for evaluating the PI have two major drawbacks. First, the evaluation of a PI requires solving a transient problem in a period long enough for the pressure to reach a PSS. When the well is not fully penetrated or directionally drilled (deviated or horizontal), the period necessary for the pressure to stabilize may become excessively long, creating difficulties for computational procedures. To address the problem of excessively long computations, some simplifying assumptions are made. Most of the methods are based on the assumption that the thickness of the reservoir is small enough to make the flow in the vertical direction negligible or so insignificant that its impact on the distribution of pressure can be included in a skin factor $[20,11]$. With the restriction on the reservoir thickness, the problem reduces to a two-dimensional one. Then the techniques for two-dimensional reservoirs can be applied. The majority of such techniques utilize the method of images, creating the second drawback - restrictions on the geometry of the domain.

With this in mind, a number of numerical experiments were conducted for various 
well configurations in three-dimensional domains. Here we illustrate the behavior of the PIs in a general homogeneous three-dimensional reservoir/well system. Equations (24) and (42) are convenient to use in such settings, since they require only solution of steady-state three-dimensional problems. Note that the use of (24) implies that in a constant rate of production regime, the pressure is uniformly distributed on the wellbore at each $t>0$. One can argue that this assumption is physically realistic for horizontal wells of any length, if we assume that the wellbore has infinite conductivity so that the pressure of the fluid entering the wellbore instantly equalizes at every point of the wellbore. For vertical or slanted wells, the assumption of uniform pressure distribution on the wellbore at each $t>0$ implies that we neglect gravity effects. Certainly, for long vertical or slanted wells, this assumption is not physically realistic.

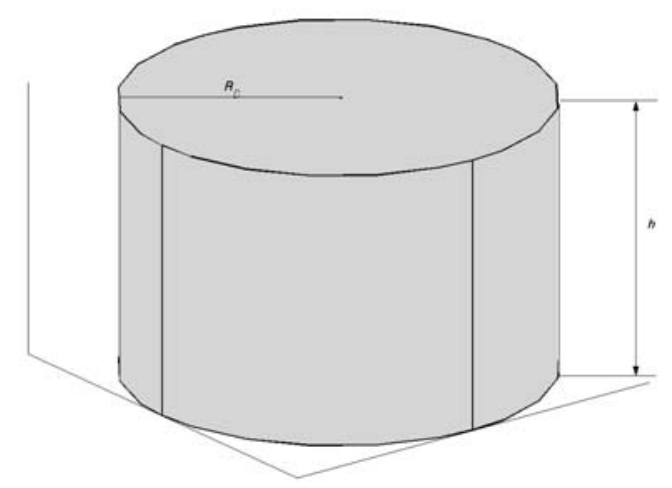

FIG. 5. Schematic representation of domain $D_{1}$.

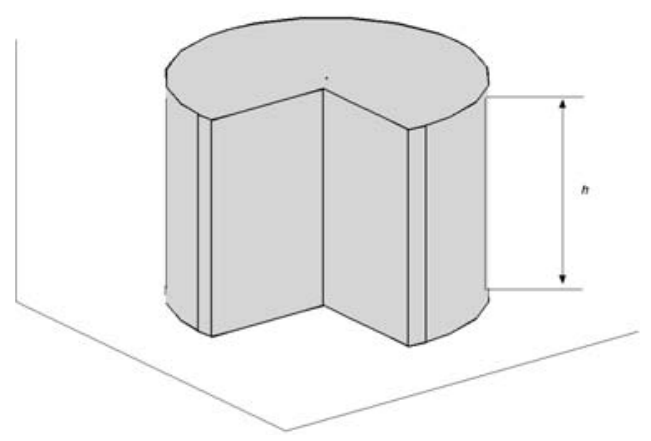

FIG. 6. Schematic representation of domain $D_{2}$.

Two domains modeling three-dimensional reservoirs that were considered for the numerical study are depicted in Figures 5, 6, and 7. Domain $D_{1}$ is a cylindrical reservoir of uniform thickness $h$ and the dimensionless radius $R_{D}$. Analogously to the two-dimensional definition, $R_{D}$ is defined as the ratio of the radius of the horizontal cross-section (in this case, circle) to the well radius. The value of $R_{D}$ is set to 1000 for all settings. For consistency of comparisons made below, the radius of the circle 


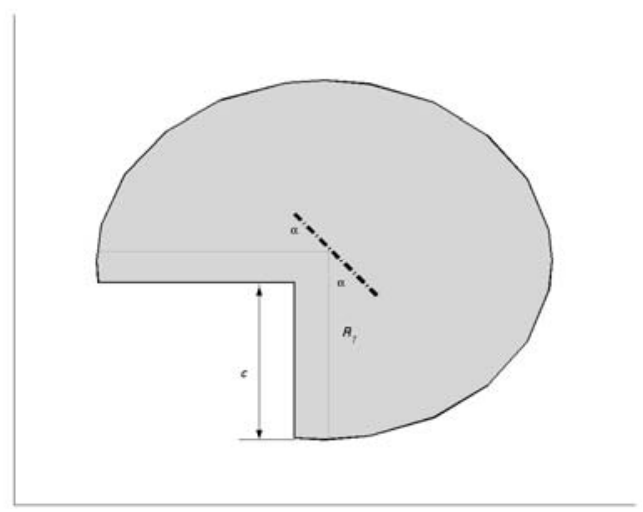

FIG. 7. Schematic representation of horizontal projection of domain $D_{2}$.

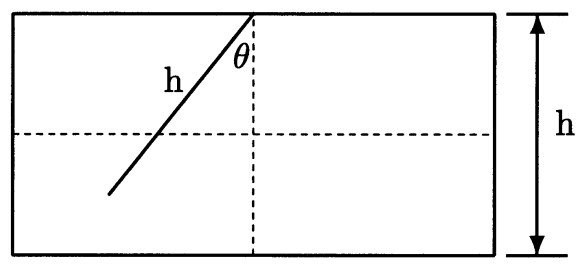

FIG. 8. Schematic representation of the vertical cross-section for well configuration (C).

of the cross-section of the domain $D_{2}$ is chosen so that the remaining area is equal to the area of the cross-section of domain $D_{1}$; i.e., the dimensionless radius associated with the horizontal cross-section of $D_{2}$ is $R_{D}=1000$.

Two well configurations were considered for both reservoir models. For domain $D_{2}$, the direction of any considered well was such that its projection on the top of the reservoir corresponded to the schematic configuration shown in Figure 7. A well is modeled by a circular cylinder with the dimensionless radius $r_{w}=1$. Then for both domains $D_{1}$ and $D_{2}$, the cross-section by the plane containing the well is a rectangle. Figures 8 and 9 show such cross-sections for every well configuration considered in the computational experiments. In configuration $(E)$, the center of symmetry of the well coincides with the center of symmetry of the cross-section. In configuration $(C)$, the well is drilled from the middle of the top side of the reservoir cross-section.

7.1. Directionally drilled wells. Effect of vertical flow. Productivity indices for well configuration (C) for domains $D_{2}$ and $D_{1}$ are given in Tables 2 and 3, respectively. In all cases, the penetration length of the well is equal to $h$ so that for $\theta=0$, the vertical well fully penetrates the reservoir. The graphs of $J_{\mathrm{I}}$ and $J_{\mathrm{II}}$ as functions of the angle $\theta$ of the well direction, shown in Figures 10 and 11, reveal that the optimal direction of a well of the fixed penetration length is not the vertical one. It is a clear indication of the effect of the vertical flow of fluid from the bottom of the reservoir toward the slanted well. This effect cannot be quantified by a reduced two-dimensional problem for a fully penetrated vertical well.

7.2. Horizontal well. Methods presented in $[20,11]$ rely heavily on the assumption that the vertical dimension of the reservoir is small compared to the penetration 


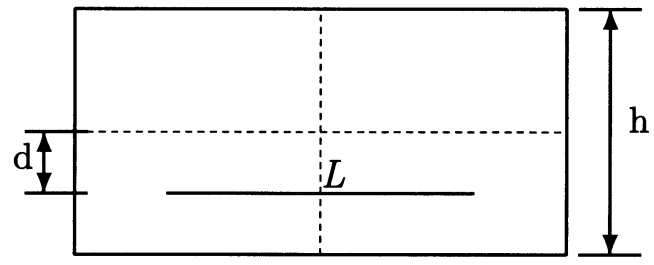

FIG. 9. Schematic representation of the vertical cross-section for well configuration (E).

TABLE 2

PIs for domains $D_{2}$, well configuration (C).

\begin{tabular}{|l||c|c|c|c|c|c||}
\hline$\theta$ & 0 & 15 & 30 & 45 & 60 & 75 \\
\hline$J_{\text {I }}$ & 0.1597 & 0.1714 & 0.1673 & 0.1634 & 0.1586 & 0.1529 \\
\hline$J_{\text {II }}$ & 0.1587 & 0.1704 & 0.1662 & 0.1623 & 0.1576 & 0.1520 \\
\hline $\begin{array}{l}\mid \frac{J_{I}-J_{\text {II }}}{J_{\text {II }}} \\
\text { percent }\end{array}$ & 0.60 & 0.64 & 0.64 & 0.67 & 0.61 & 0.59 \\
\hline
\end{tabular}

TABLE 3

PIs for domain $D_{1}$, well configuration (C).

\begin{tabular}{|c||c|c|c|c|c|c|c|c||}
\hline & $\theta$ & 0 & 8 & 15 & 30 & 45 & 60 & 75 \\
\hline \multirow{3}{*}{$h=100$} & $J_{\mathrm{I}}$ & 0.1629 & 0.1705 & 0.1765 & 0.1718 & 0.1691 & 0.1680 & 0.1662 \\
\cline { 2 - 9 } & $\begin{array}{c}J_{\text {II }} \\
\frac{J_{I}-J_{\text {II }}}{J_{\text {II }}} \\
\text { percent }\end{array}$ & 0.1623 & 0.1696 & 0.1758 & 0.1710 & 0.1683 & 0.1672 & 0.1655 \\
\hline \hline \multirow{2}{*}{$h=200$} & $J_{\text {I }}$ & 0.1629 & 0.1665 & 0.1697 & 0.1611 & 0.1426 & 0.1315 & 0.1199 \\
\cline { 2 - 9 } & \begin{tabular}{c}
$J_{\text {II }}$ \\
\cline { 2 - 8 } \\
$\frac{J_{I}-J_{\text {II }}}{J_{\text {II }}}$ \\
percent
\end{tabular} & 0.1623 & 0.1658 & 0.1689 & 0.1605 & 0.1422 & 0.1312 & 0.1196 \\
\hline
\end{tabular}

length of the well. Moreover, as noted in [20], the precision of the evaluation of the PI for horizontal wells decreases drastically as the distance from the well to vertical boundaries of the reservoir becomes comparable to the distance to the top and/or the bottom of the reservoir, if the reduction to the two-dimensional problem is used. This section presents computational results for such settings when the assumption of the small reservoir thickness and the well being clearly inside the drainage area are relaxed.

The setting considered is a horizontal well with configuration $(\mathrm{E})$, located at distance $d$ below the plane of symmetry of domain $D_{1}$. The graphs of the computed PSS PI $J_{\mathrm{I}}$ as a function of distance $d$ from the center of the reservoir for various penetration lengths $L$ are shown in Figure 12.

For all practical purposes, one can conclude that the optimal location of a horizontal well in a cylindrical reservoir $D_{1}$ is in the horizontal plane of symmetry of the reservoir. Note that for long wells, however, the PSS PI slightly increases for small values of $d$. This may be an indication of an interesting feature of the diffusive capacity as a geometrical characteristic defined through the first eigenvalue $\lambda_{0}$. The latter is sensitive to the location of the well relative to the planes and lines of symmetry of the domain, as it is comprehensively illustrated in section 6 . In three-dimensional 


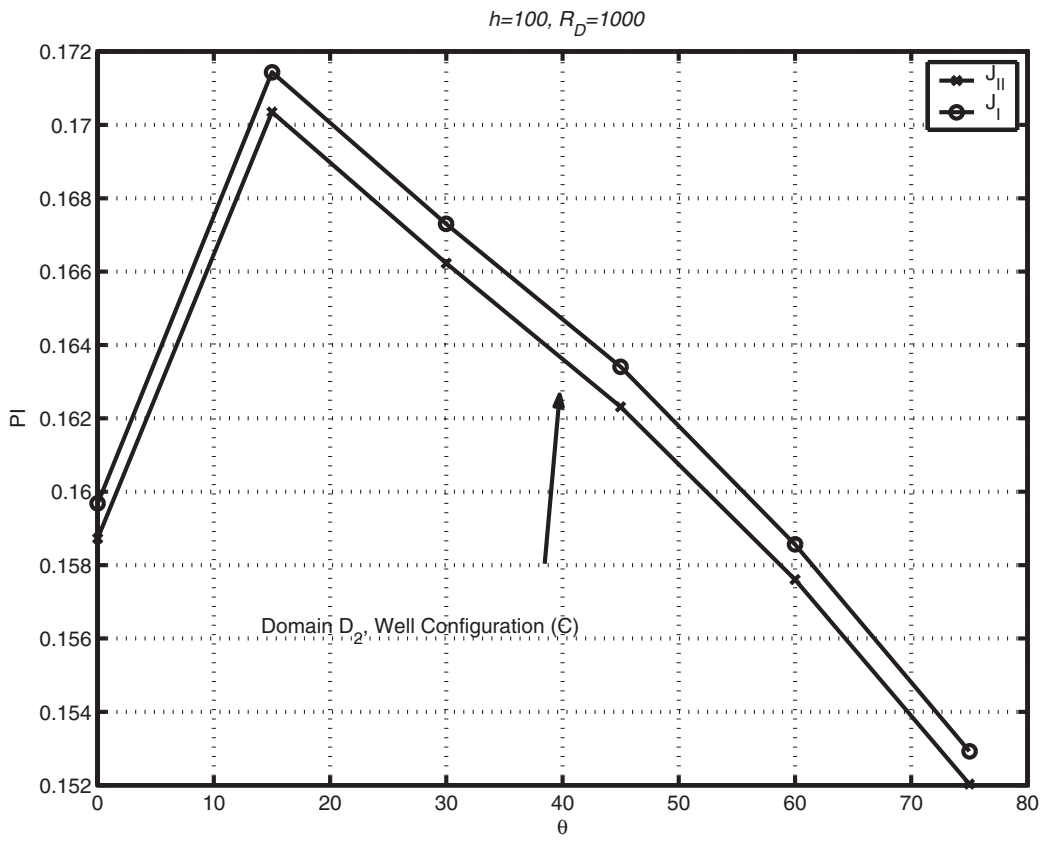

FIG. 10. PIs for domain $D_{2}$, well configuration (C).

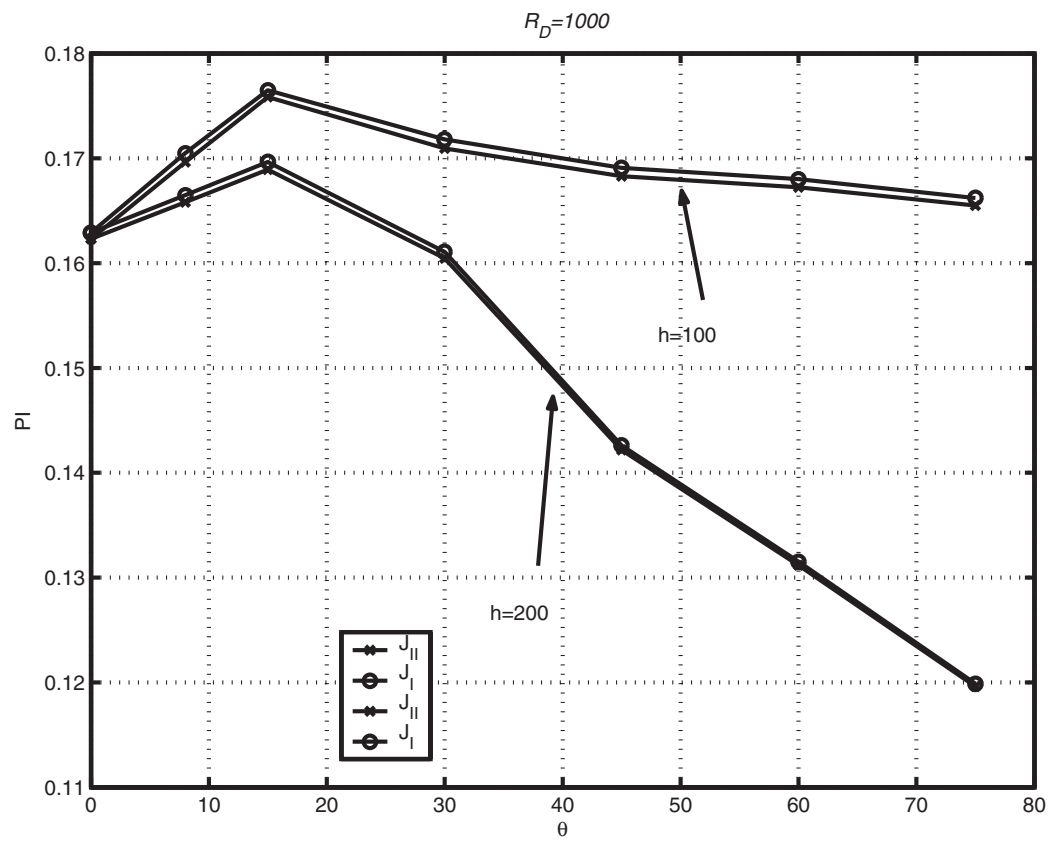

FIG. 11. PIs for domain $D_{1}$, well configuration (C).

domains, there are more such planes and lines of symmetry and, therefore, there may be several well configurations yielding maximal PI. 

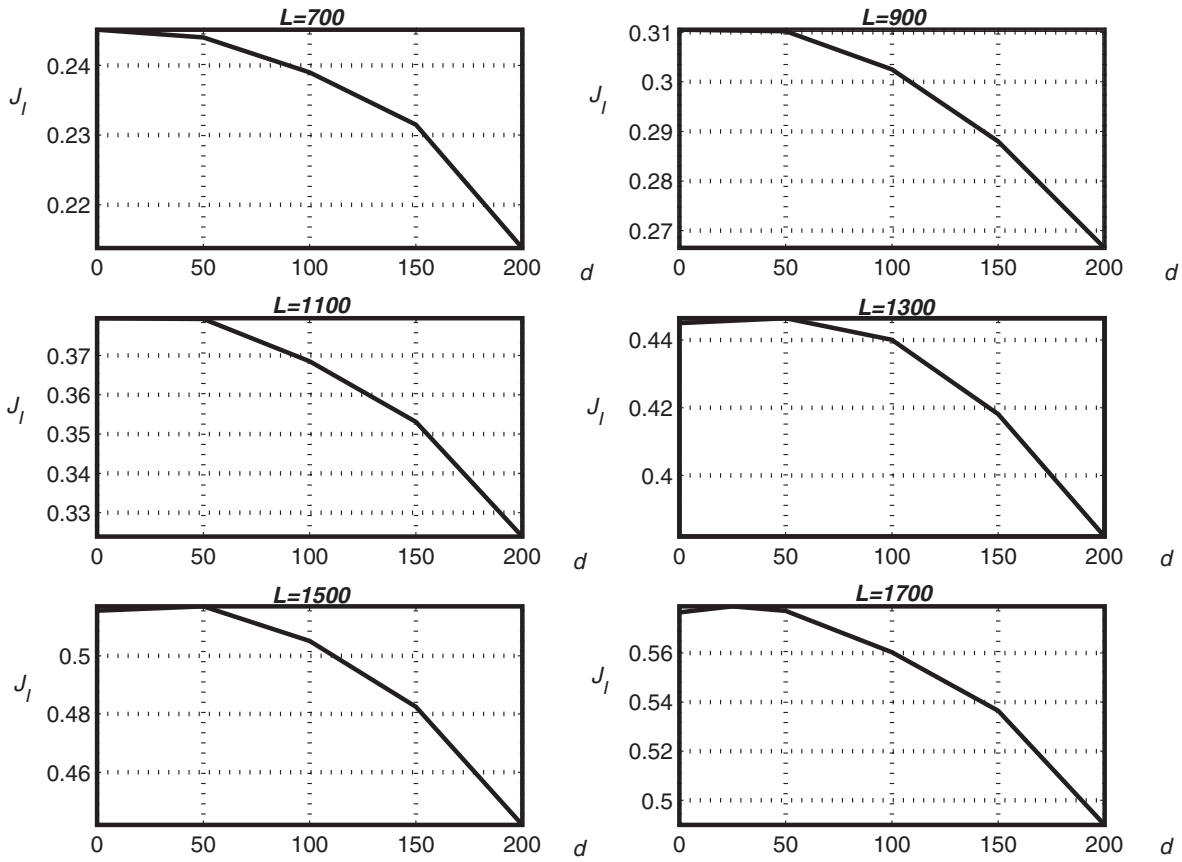

FIG. 12. PSS PI for various values of $d$ and $L$, well configuration (E), $h=500$.

\section{REFERENCES}

[1] R. A. Adams, Sobolev Spaces, Academic Press, New York, 1975.

[2] T. A. Blasingame, L. E. Doublet, and P. P. Valko, Development and application of the multiwell productivity index (MPI), SPE J., 5 (2000), pp. 21-31.

[3] H. Cinco-Ley, H. J. Ramey, Jr., and F. G. Miller, Pseudo-skin factors for partially penetrating directionally drilled wells, in Proceedings of the 50th Annual Fall Meeting, SPE 5589, Dallas, TX, 1975.

[4] H. Cinco, F. G. Miller, And H. J. Ramey, JR., Unsteady-state pressure distribution created by a directionally drilled well, J. Petroleum Technology, Nov. 1975, pp. 1392-1400.

[5] R. Courant and D. Hilbert, Methods of Mathematical Physics, Vol. 1, Interscience Publishers, New York, 1953.

[6] D. N. Dietz, Determination of average reservoir pressure from build-up surveys, J. Petroleum Technology, Aug. 1965, pp. 955-959.

[7] R. C. Earlougher, Jr., H. J. Ramey, Jr., F. G. Miller, and T. D. Mueller, Pressure distributions in rectangular reservoirs, J. Petroleum Technology, Feb. 1968, pp. 200-208.

[8] M. J. Fetkovich, The isochronal testing of oil wells, in Proceedings of the 48th Annual Fall Meeting, SPE 4529, Las Vegas, NV, 1973.

[9] M. J. Fetkovich, E. J. Fetkovich, and M. D. Fetkovich, Useful concepts for decline-curve forecasting, reserve estimation and analysis, SPE Reservoir Engrg., Feb. 1996, pp. 13-22.

[10] A. Friedman, Partial Differential Equations of Parabolic Type, Prentice-Hall, Englewood Cliffs, NJ, 1964.

[11] P. A. Goode And F. J. Kuchuk, Inflow performance of horizontal wells, SPE Reservoir Engrg., Aug. 1991, pp. 319-323.

[12] M. F. Hawkins, JR., A note on the skin effect, Petr. Trans. AIME, 207 (1956), pp. 356-357.

[13] W. Helmy and R. A. Wattenbarger, New shape factors for wells produced at constant pressure, in Proceedings of the SPE Gas Technology Symposium, SPE 39970, Calgary, Canada, 1998.

[14] W. Hurst, Establishment of skin effect and its impediment to fluid flow into a well bore, Petroleum Engineer, 25 (1953), pp. B-6-B-16. 
[15] W. Hurst, J. D. Clark, and E. B. Brauer, The skin effect in producing wells, J. Petroleum Technology, Nov. 1969, pp. 1483-1489.

[16] A. Ibragimov and P. VAlKo, On productivity index in pseudo-steady and boundary dominated flow regimes, Technical report, Institute of Scientific Computations, 2000; also available online from http://www.isc.tamu.edu/iscpubs/iscreports.html.

[17] A. I. Ibragimov And M. N. Baganova, Study of transient flow filtration towards a single horizontal well, in Fundamental Bases of New Technologies in Oil and Gas Industry, Nauka, Moscow, 2000, pp. 192-198.

[18] A. I. Ibragimov and E. M. Landis, On the behavior of the solution of the Zaremba problem in the neighborhood of the boundary point and at the infinity, Dokl. Math., 57 (1998), pp. $185-186$.

[19] R. F. KRUEGer, An overview of formation damage and well productivity in oilfield operations, J. Petroleum Technology, Feb. 1986, pp. 131-152.

[20] L. LARSen, General productivity models for wells in homogeneous and layered reservoirs, in Proceedings of the SPE Annual Conference and Exhibition, SPE 71613, New Orleans, LA, 2001.

[21] C. S. Matthews, F. Brons, and P. Hazebroek, A method for determination of average pressure in a bounded reservoir, Trans. AIME, 201 (1954), pp. 182-191.

[22] V. G. Maz'ya, Differentiable Functions on Bad Domains, World Scientific, Singapore, 1997.

[23] M. Muskat, The Flow of Homogeneous Fluid Through Porous Media, McGraw-Hill, New York, 1937.

[24] L. E. Payne and I. Stakgold, On the mean value of the fundamental mode in the fixed membrane problem, Appl. Anal., 3 (1973), pp. 225-303.

[25] J. K. Pucknell and P. J. Clifford, Calculations of total skin factors, in Proceedings of the Offshore Europe Conference, SPE 23100, Aberdeen, UK, 1991.

[26] R. Raghavan, Well Test Analysis, Prentice-Hall, Englewood Cliffs, NJ, 1991.

[27] H. J. Ramey, JR., And W. M. CoBb, A general pressure buildup theory for a well in a closed drainage area, J. Petroleum Technology, Dec. 1971, pp. 1493-1505.

[28] D. J. Romero, P. P. Valko, and M. J. Economides, Optimization of the productivity index and the fracture geometry of a stimulated well with fracture face and choke skins, SPE Prod. Facilities, 18 (2003), pp. 57-63.

[29] T. YILDIZ, Assessment of total skin factors in perforated wells, in Proceedings of the SPE European Formation Damage Conference, SPE 82249, Hague, The Netherlands, 2003.

[30] A. F. VAN EVERDINGEN, The skin effect and its influence on the productivity capacity of a well, Petroleum Trans. AIME, 198 (1953), pp. 171-176. 